\title{
DESDOBRAMENTOS DA TOMADA DE DECISÃO EM PROBLEMAS DE SUBSTITUIÇÃO DE EQUIPAMENTOS POR MEIO DE FUNÇÕES CONTÍNUAS E ANÁLISE NÃO SUAVE
}

\section{DEVELOPMENTS ON DECISION-MAKING OF EQUIPMENT REPLACEMENT PROBLEMS THROUGH CONTINUOUS FUNCTIONS AND NON- SMOOTH ANALYSIS}

\author{
Igor Gimenes Cesca* E-mail: igcesca@gmail.com \\ *IBMEC, SP e IEE/USP, São Paulo, SP
}

\begin{abstract}
Resumo: O problema de substituição de equipamentos é analisado tradicionalmente por duas metodologias discretas: programação dinâmica e indicadores de fluxo de caixa. Constatou-se que essas metodologias não conseguem verificar se (i) o momento ótimo de substituição de equipamentos é único e (ii) se existiria algum ativo físico que não precisasse ser substituído, isto é, um equipamento sem momento ótimo de substituição do ponto de vista econômico. Sendo assim, os autores utilizaram neste trabalho uma abordagem com funções contínuas para resolver esse tipo de problema. No processo de estabelecê-las, chegou-se em funções não diferenciáveis (no sentido clássico). Logo, foi utilizada uma análise não suave para modelagem. Pelos resultados obtidos é possível afirmar que de fato o momento ótimo de substituição é único. Além disso, também verificou-se que pode existir equipamentos sem momento ótimo para substituição. Mais ainda, também foi viável formular um modelo para calcular o momento ótimo de substituição de equipamentos por função contínua, conforme são dados os custos de manutenção, o valor de aquisição, a taxa de desvalorização e a taxa de desconto.
\end{abstract}

Palavras-chave: Vida econômica de ativos físicos. Reposição. Custo de ciclo de vida. Engenharia econômica. Gestão de ativos físicos. Manutenção.

\begin{abstract}
The problem of equipment replacement is traditionally analyzed by two discrete methodologies: dynamic programming and cash flow indicators. It was noticed that these methodologies cannot verify if (i) the optimal moment of equipment replacement is unique and (ii) if there were any physical assets that did not need to be replaced, that is, from the economical point of view, an equipment without optimal moment of substitution. Accordingly, in this work, the authors used an approach with continuous functions to solve this type of problem. In the process of establishing these functions, we obtained non-differentiable functions (in the classical sense). Therefore, a non-smooth modeling analysis was used. From the obtained results, it is possible to affirm that the optimal moment of substitution is unique. It has also been found that there may be equipment with no optimum moment for replacement. Moreover, it was also possible to formulate a model to calculate the optimum moment of equipment substitution by continuous function, according to the maintenance costs, the acquisition value, the depreciation rate and the discount rate.
\end{abstract}

Keywords: Economic life of physical assets. Replacement. Life-cycle cost. Engineering economic. Physical assets management. Maintenance. 


\section{INTRODUÇÃO}

Os problemas de substituição de equipamentos, os quais envolvem a determinação do momento ótimo de reposição de ativos físicos e também a determinação de vida econômica, são clássicos na engenharia de produção e na econômica, oriundos da década de 1950. Uma abordagem na resolução desses problemas é utilizar algoritmos de programação dinâmica, segundo os autores Bellman (1955), Waddel (1983), Nakagawa (1984), Hartman (2004), Abensur (2015) e Cesca (2017), enquanto outra é utilizar indicadores de fluxo de caixa de acordo com Alchian (1958), Longo; Zaboneti (1989), Sullivan et al (2002) e Dhillon (2010). Por ambas as técnicas, as premissas do problema-como, por exemplo, tempo, custo de aquisição e custos de manutenção - são modeladas por modelos matemáticos discretos.

Entretanto, por meio de uma revisão de literatura feita pelos autores deste trabalho, percebeu-se que a minoria dos trabalhos sobre esta temática utilizou modelos matemáticos contínuos. Em geral, quando foi feito uso de uma abordagem contínua, isso ocorreu considerando custos de manutenção e desvalorização do ativo estocástico e modelado por movimento geométrico browniano, como por exemplo, em Ye (1990), Mauer e Ott (1995), Fernandez (2001), Zambujal-Oliveira e Duque (2011) e, mais recentemente, em Yatsenko e Hritonenko (2017) e Riechi et al (2017)

Ainda na pesquisa de trabalhos sobre esse tema na literatura, os autores deste trabalho levantam alguns questionamentos sobre o estado de arte que envolve o problema de substituição de equipamentos. Percebeu-se que nos trabalhos encontrados, em relações às premissas clássicas dos modelos substituição de equipamentos, há lacunas sobre as condições de existências e unicidades dos pontos de vida econômica e momentos ótimos de reposição de ativos.

Mais especificamente, (1) não foram encontrados, na literatura, modelos que determinam se há mais de um ponto de vida econômica, isto é, não foi demonstrado se é possível existir mais de um momento ótimo de substituição. Não só, mas também (2) questiona-se se seria possível existir algum ativo físico que nunca será substituído, isto é, sua curva de custo de propriedade não atinge um ponto mínimo, o qual implica na vida econômica, ou seja, o momento ótimo de substituição. Sendo assim, se conjectura se, com um tratamento matemático mais sofisticado, seria possível responder os questionamentos levantados pela revisão bibliográfica. 


\section{REFERENCIAL TEÓRICO}

Para uma empresa, um ativo físico é um bem. Logo, os principais ativos físicos são máquinas, prédios, veículos, equipamentos, sistemas de software. Para o funcionamento desses ativos não serem comprometidos são necessárias manutenções de caráter preditivo, preventivo e também, quando necessário, corretivo, as quais apresentam custos. O conhecimento desses custos é importante, uma vez que, com o passar do tempo, esses podem aumentar e equivaler a várias vezes 0 valor do custo de aquisição do ativo (BROWN; YANUCK, 1985). Dessa forma, a aquisição de um ativo não deve ser decidida mediante apenas ao seu custo de aquisição, mas por meio de um estudo que leve em conta as despesas de manutenção ao longo de toda sua vida útil inclusive (DHILLON, 2010).

Se um ativo for mantido por um período longo, as despesas de manutenção terão valores muito elevados. Por outro lado, se o ativo for trocado prematuramente, o custo de capital não terá sido amortizado. Logo, parte do investimento na aquisição do ativo será perdida (MARQUES, 2003), (ADKINS; PAXSON, 2013), (CESCA, 2017). Sendo assim, convém determinar o momento ideal de troca de equipamentos. Portanto, surge o conceito de vida econômica, o qual é o período ótimo de uso do ativo.

O procedimento tradicional para encontrar a vida econômica se dá por meio de indicadores de fluxo de caixa. De acordo com Ross et al (2002), a demonstração em fluxo de caixa ajuda a explicar a variação dos saldos de caixas e aplicações financeiras. Esta metodologia é utilizada para facilitar a avaliação do impacto das entradas e saídas de caixa em diferentes anos. O critério mais utilizado consiste na Anuidade Equivalente (AE), também chamado de Custo Anual Uniforme Equivalente. A proposta deste critério baseia-se em converter os valores de um fluxo de caixa irregular em uma série de pagamentos uniformes idênticos. Assim, o valor de anuidades equivalentes informa o quanto é gasto por ano com o ativo. Este critério é calculado por meio da equação (1).

$$
A E(n)=V P L *\left[\frac{i *(1+i)^{n}}{(1+i)^{n}-1}\right]
$$

$\mathrm{Na}$ equação (1), $i$ representa a taxa mínima de atratividade, $n$ é o prazo de duração do projeto e VPL (valor presente líquido) abarca o somatório das entradas e saídas de caixas descontadas para a data zero pela taxa $i$.

Revista Produção Online. Florianópolis, SC, v. 18, n. 3, p. 850-874, 2018. 
Com o critério anuidade equivalente fundamentado, definem-se alguns conceitos: custo equivalente de capital, custo equivalente de manutenção e, por último, custo equivalente de propriedade. Todos esses conceitos, baseados em Park (2002), foram utilizados para a metodologia deste artigo na seção seguinte.

- Custo equivalente de capital (CEC): O custo de capital possui duas componentes: custo de aquisição $(\mathrm{CA})$ e valor residual $(V R(n))$ do ativo, sendo que o primeiro possui um valor fixo e o segundo é variável em relação ao tempo. Calcula-se a diferença das anuidades equivalentes de cada uma das componentes, conforme a equação (2).

$$
\operatorname{CEC}(n)=\left(\frac{i *(1+i)^{n}}{(1+i)^{n}-1}\right)\left(C A-\sum \frac{V R(n)}{(1+i)^{n}}\right)
$$

- Custo equivalente de manutenção (CEM): Para encontrar o custo equivalente de manutenção, primeiramente é necessário calcular o valor presente do somatório da série de custos de manutenção $(C M(n))$ ao longo do tempo. Feito isso, calculase a anuidade equivalente do valor encontrado, conforme é possível ver na equação (3).

$$
\operatorname{CEM}(n)=\left(\frac{i *(1+i)^{n}}{(1+i)^{n}-1}\right)\left(\sum \frac{C M(n)}{(1+i)^{n}}\right)
$$

- Custo equivalente de propriedade (CEP): O custo equivalente de propriedade de um ativo é a soma dos custos de capital e de manutenção em anuidades equivalentes, ou seja, a soma das funções $C E M(n)$ e $C E C(n)$ conforme é possível ver na equação (4).

$$
C E P(n)=\left(\frac{i *(1+i)^{n}}{(1+i)^{n}-1}\right)\left[\left(C A-\frac{V R(n)}{(1+i)^{n}}\right)+\sum \frac{C M(n)}{(1+i)^{n}}\right]
$$

É possível ver na Figura 1 uma representação gráfica de como se comportam as curvas. 
Figura 1 - Modelo para estimativa de vida econômica

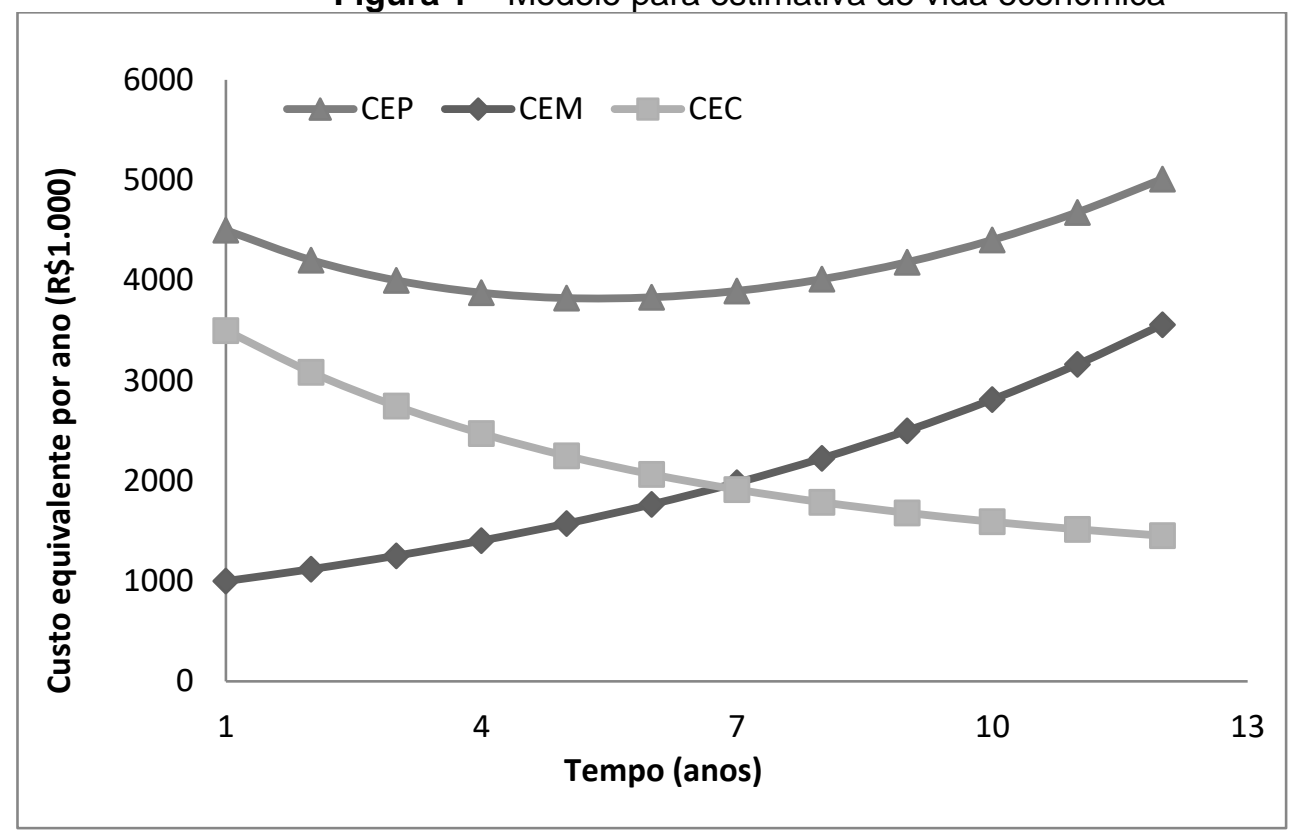

Fonte: Os autores

Na Figura 1 encontram-se os custos (no eixo vertical) das três variáveis ao longo do tempo (no eixo horizontal). É possível ver, o custo equivalente de manutenção aumenta com o tempo, enquanto o custo equivalente de capital diminui, sendo que a soma de ambas as curvas resulta na curva de custo equivalente de propriedade do ativo. Nesta curva, o ponto de valor mínimo representa o período ótimo de uso do ativo; isto é, a vida econômica do ativo, a qual, para o exemplo é de cinco anos. Assim, este é o período ótimo em que um ativo deve ser utilizado.

Apresentado este referencial teórico, os autores colocaram algumas questões que surgiram ao longo dessa pesquisa bibliográfica, tais como: (1) O ponto de vida econômica é único? Isto é, seria possível existir mais de um ponto de vida econômica na curva de custo de propriedade? (2) Quais as condições para existir um ponto de vida econômica? (3) É possível encontrar um ponto de vida econômica para todos os ativos físicos?

\section{PROCEDIMENTOS METODOLÓGICOS}

Para responder as questões levantadas no final da seção anterior, os conceitos anteriormente apresentados serão formalizados por funções matemáticas contínuas, a fim de que haja mais rigor por meio de fundamentos do cálculo para responder tais 
questões. Estas funções não são do tipo diferenciáveis (no sentido clássico), demandando uma análise não suave.

Dessa maneira, os seguintes modelos são formalizados:

- Curva de custo equivalente de capital, conforme mostra a equação (5).

$$
C E C=\left(\frac{e^{r}-1}{e^{r t}-1} e^{r t}\right)\left(A-R(t) * e^{-r t}\right)
$$

- Curva de custo equivalente de manutenção, conforme mostra a equação (6).

$$
C E M=\left(\frac{e^{r}-1}{e^{r t}-1} e^{r t}\right) \int_{0}^{t}\left(M(t) * e^{-r t}\right) d t
$$

Nas quais:

- $\left(\frac{e^{r}-1}{e^{r t}-1} e^{r t}\right)$ é o fator de equivalência de fluxo de caixa presente em anuidades regulares para o caso contínuo. Além disso, $r$ é a taxa de juros, tal que $0 \leq r \leq$ 1.

- $M(t)$ representa os gastos de manutenção ao longo do tempo. Essa função pode ser encontrada por meio de regressão linear a partir de coleta de dados de custos de manutenções de ativos. Além disso, é aceitável que $M(t)$ seja linear e crescente, uma vez que um dos pressupostos dos custos de manutenção é que estes sejam maiores a cada ano. Por isso, define-se $M(t)=a t$, com $a>0$.

Entretanto, $M(t)$ pode assumir outras formas, sem perda de generalidade para as equações, uma vez que a integral $\int_{0}^{t}\left(M(t) * e^{-r t}\right) d t$ representa a transformada de Laplace da função $M(t)$ (BUSER, 1986).

- $A$ representa o custo de aquisição do ativo;

- $R(t)$ é o valor de revenda do equipamento no ano de substituição $t$. Uma vez que ativos são vulneráveis à depreciação física, espera-se que $R(t)$ seja decrescente. Contudo, $R(t)$ é sempre positiva. Afinal, é impossível o ativo ter valor negativo. Assim, a função é definida da seguinte forma:

$$
R(t)=\left\{\begin{array}{cc}
A-b t & , 0 \leq t<A / b \\
0 & , t \geq A / b
\end{array} \text {, tal que } b\right. \text { é a taxa de desvalorização do ativo a }
$$
cada ano e $b>0$ e $A>0$. 
Em relação ao comportamento da função $R(t)$, percebe-se que não se trata de uma função analítica. $O$ motivo da escolha desta função para modelar o valor de renda de ativos físicos foi que com esta função fica determinada a função de vida econômica para o caso mais difícil de modelar. Caso se encontre empiricamente alguma outra função de valor de revenda analítica, a modelagem se torna mais fácil. De qualquer forma, a formulação sugerida aqui neste artigo, modela ambos os casos de a função $R(t)$ ser ou não analítica.

Assim, o custo equivalente de propriedade (CEP) fica formalizado da seguinte forma:

$$
C E P=C E M+C E C=\left[\frac{\left(e^{r}-1\right) * e^{r t}}{e^{r t}-1}\right] *\left(\int_{0}^{t}\left(M(t) * e^{-r t}\right) d t+A-R(t) * e^{-r t}\right)
$$

Logo, define-se a equação (7):

$$
A E(t)=\left[\frac{\left(e^{r}-1\right) * e^{r t}}{e^{r t}-1}\right] *\left(\int_{0}^{t}\left(M(t) * e^{-r t}\right) d t+A-R(t) * e^{-r t}\right)
$$

Ocorre que, dado o comportamento de $R(t)$, o qual não é diferenciável em $t=$ $A / b$, convém analisar o modelo na equação (7) em duas partes, sendo o Regime 1 , $0 \leq t<A / b$, e o Regime 2, $t \geq A / b$. Dessa maneira, a equação (7) é reescrita da seguinte maneira:

$$
\begin{aligned}
& A E(t) \\
& = \begin{cases}{\left[\frac{\left.e^{r}-1\right) * e^{r t}}{e^{r t}-1}\right] *\left(\int_{0}^{t}\left(M(t) * e^{-r t}\right) d t+A-R(t) * e^{-r t}\right)} & , 0 \leq t<A / b \\
{\left[\frac{\left(e^{r}-1\right) * e^{r t}}{e^{r t}-1}\right] *\left(\int_{0}^{t}\left(M(t) * e^{-r t}\right) d t+A\right)} & , t \geq A / b\end{cases}
\end{aligned}
$$

Sendo a primeira parte referente ao Regime 1 e a segunda ao Regime 2.

É importante comparar a equação (1) com a (7). Na primeira, é calculada a anuidade equivalente em função de $n$ de forma discreta. Sendo assim, $A E(n)$ não está definida para $n=0$. Já na segunda equação, $A E(t)$ é contínua. Dessa maneira, embora haja um "salto" em $t=0$, como $\lim _{t \rightarrow 0} A E(t)=A E(0)$, então $A E(t)$ é contínua em $t=0$. Além disso, é importante observar que $\lim _{t \rightarrow \frac{A^{+}}{b}} A E(t)=\lim _{t \rightarrow \frac{A}{b}} A E(t)$. Portanto, a função é contínua em $t=A / b$. 


\subsection{Modelagem no Regime 1}

Nesta seção estabeleceu-se uma análise para a equação de anuidade equivalente para o Regime 1, ou seja, para quando $0 \leq t<A / b$. Assim, simplificando a equação (8) referente ao Regime 1, obteve-se a seguinte expressão, definida pela equação (9):

$$
A E(t)=\left(\frac{\left(e^{r}-1\right)}{r^{2}}\right)\left(\frac{r t(b r-a)}{e^{r t}-1}+a+A r^{2}\right)
$$

Em seguida, derivou-se a função para encontrar os pontos críticos e depois avaliar se estes são pontos de mínimo, conforme é possível observar na equação (10).

$$
\frac{d A E}{d t}(t)=\left(\frac{\left(e^{r}-1\right)}{r\left(e^{r t}-1\right)^{2}}\right)\left(e^{r t}(r t-1)+1\right)(a-b r)
$$

Nesse caso, há duas situações em que $\frac{d A E}{d t}(t)=0$ :

(1) $(a-b r)=0$.

Esta solução implica em $A E^{\prime}(t)=0$, para qualquer $t$ pertencente ao domínio de $A E$. Logo, qualquer $t$ no domínio de $A E$ é ponto crítico. Contudo, para um ponto crítico $A E\left(t_{0}\right)$ ser avaliado como mínimo de uma função é necessário que ocorra $A E^{\prime \prime}\left(t_{0}\right)>0$ e $A E^{\prime}\left(t_{0}\right)=0$. Como neste caso $A E^{\prime \prime}(t)=0$, é possível concluir que esta situação não é válida como mínimo da função $A E(t)$ em problemas de vida econômica.

(2) $\left(e^{r t}(r t-1)+1\right)=0$

Nesta situação, ocorre que $\left(e^{r t}(r t-1)+1\right)=0$, quando $t=0$. Contudo, quando se analisa a equação (10) por completo, tem-se $\frac{0}{0}$, ou seja, uma indeterminação.

Posteriormente, prosseguiu-se o estudo da função $A E(t)$ no Regime 1, analisando se ela possuía comportamento crescente, decrescente ou constante neste intervalo. Sendo assim, foi possível reescrever a equação (9) de acordo com a seguinte forma:

$$
A E(t)=\left(\frac{\left(e^{r}-1\right)}{r^{2}}\right)\left(a+A r^{2}\right)+\left(\frac{\left(e^{r}-1\right)}{r^{2}}\right)\left(\frac{r t(b r-a)}{e^{r t}-1}\right)
$$

Depois, foram definidas as seguintes funções $f(t)$ e $g(t)$ no intervalo de $0 \leq$ $t<A / b$ : 


$$
\begin{gathered}
f(t)=\left(\frac{\left(e^{r}-1\right)}{r^{2}}\right)\left(a+A r^{2}\right) \\
g(t)=\left(\frac{\left(e^{r}-1\right)}{r^{2}}\right)\left(\frac{r t}{e^{r t}-1}\right)(b r-a)
\end{gathered}
$$

Tais que,

$$
A E(t)=f(t)+g(t)
$$

Na equação (11), $f(t)$ é sempre constante para qualquer valor de $t$. Já na equação (12), por sua vez, $g(t)$ varia conforme o valor de $t$. Logo, convém analisar o comportamento desta última função. Para analisar o comportamento de uma função, convém sempre derivá-la, conforme evidenciado na equação (14).

$$
\frac{d g}{d t}(t)=\frac{\left(e^{r}-1\right) r}{r^{2}}(a-b r)\left(e^{r t}(r t-1)+1\right)
$$

Ocorre que o termo $\left(e^{r t}(r t-1)+1\right)$ é sempre positivo para $t \geq 0$. Sendo assim, o comportamento de $g(t)$ depende exclusivamente de $(a-b r)$, tal que $b$ é a taxa de desvalorização do ativo, $r$ a taxa de desconto utilizada e a o coeficiente da função de custos de manutenção $M(t)$. Dessa maneira, se $b r=a$, ou se $b r>a$, ou se $b r<a$, a função $g(t)$ possui o seguinte comportamento, conforme pode ser observado em (15):

$$
\begin{cases}b r=a, & g(t) \rightarrow \text { constante } \\ b r>a, & g(t) \rightarrow \text { decrescente } \\ b r<a, & g(t) \rightarrow \text { crescente }\end{cases}
$$

Consequentemente, como $f(t)$, na equação (11), é constante, a equação (13), da função $A E(t)$, possui o mesmo comportamento de $g(t)$. Portanto, no Regime 1, $A E(t)$ possui uma das três seguintes possíveis funções, conforme é possível ver na

Figura 2.

Figura 2 - Comportamento da função AE (t) no Regime 1
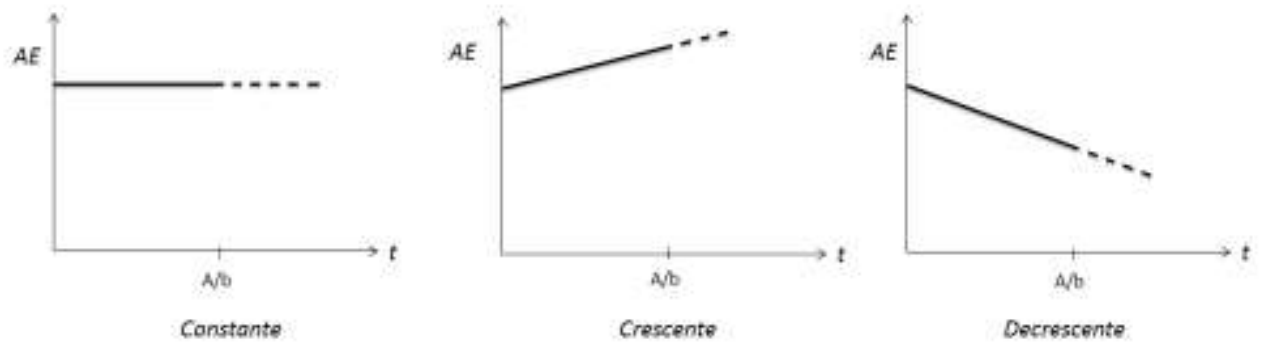

Fonte: Os autores

$\mathrm{Na}$

Revista Produção Online. Florianópolis, SC, v. 18, n. 3, p. 850-874, 2018. 
Figura 2, ficam evidenciados os possíveis comportamentos da função $A E(t)$ na região de $0 \leq t<A / b$, os quais são crescente, decrescente e constante. Contudo, o comportamento constante da função não é observável para um caso real. Ocorre que $a-b r=0$, então tem-se que, conforme a equação (16):

$$
a=b r
$$

A equação (16) é representada graficamente, conforme é possível ver na Figura 3.

Figura 3 - Representação da equação (16) no plano

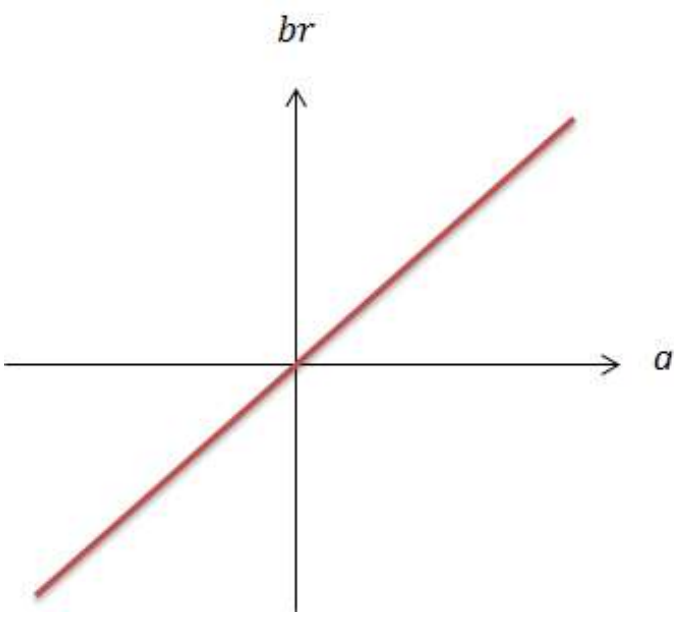

Fonte: Os autores

Na Figura 3, é possível observar o plano formado pelos eixos com os valores de $a$ e $b r$. Percebe-se que qualquer ponto acima da reta implica em $b r>a$, já qualquer ponto abaixo da reta implica em $b r<a$ e, por último, qualquer ponto na reta implica em $b r=a$.

Em um plano toda reta tem área nula, enquanto o plano tem área infinita. Logo, a chance de um ponto cair aleatoriamente na reta é nula. Por isso, a probabilidade de a função $A E(t)$ ser constante no Regime 1, para um caso real, é zero. Assim, concluise que a função $A E(t)$ no intervalo $[0, A / b$ [ possui um comportamento ou crescente ou decrescente.

\subsection{Modelagem no Regime 2}

No Regime 2, foi analisado o comportamento da função $A E(t)$ para $t>A / b$. Sendo assim, definiu-se a equação (17), simplificando a equação (8) para esta região: Revista Produção Online. Florianópolis, SC, v. 18, n. 3, p. 850-874, 2018. 


$$
A E(t)=\frac{\left(-1+e^{r}\right)}{\left(-1+e^{r t}\right) r^{2}}\left(e^{r t}\left(a+A r^{2}\right)-a(1+r t)\right)
$$

Calculando a derivada de (17) para encontrar os pontos críticos, temos que:

$$
\frac{d A E}{d t}(t)=\frac{\left(-1+e^{r}\right)}{r\left(-1+e^{r t}\right)^{2}}\left(a-e^{r t}\left(-a r t+a+A r^{2}\right)\right)
$$

Dessa maneira, a fim de facilitar o entendimento, define-se:

$$
h(t)=\left(a-e^{r t}\left(-a r t+a+A r^{2}\right)\right)
$$

tal que, $\frac{d A E}{d t}(t)=\frac{\left(-1+e^{r}\right)}{r\left(-1+e^{r t}\right)^{2}} * h(t)$

Então, como $\frac{\left(-1+e^{r}\right)}{r\left(-1+e^{r t}\right)^{2}}>0$, para qualquer valor $t$ em $t>A / b$, então se calcula $h(t)=0$, a fim de encontrar os pontos críticos da equação (18).

Sejam

$$
\tau=\operatorname{rt~e} c=\frac{A r^{2}}{a} \text {. }
$$

Dessa maneira, reescreve-se a equação (19) da seguinte maneira:

$$
h(\tau)=1-e^{\tau}(-\tau+1+c)
$$

Destarte, iguala-se a equação (20) a zero, com a finalidade específica de se identificar os pontos críticos nela.

$$
1-e^{\tau}(-\tau+1+c)=0 .
$$

Resultando em:

$$
c=\frac{1-e^{\tau}+\tau e^{\tau}}{e^{\tau}}
$$

Assim, foi possível definir a seguinte função $p(\tau)$, conforme é possível ver na equação (22):

$$
p(\tau)=\frac{1-e^{\tau}+\tau e^{\tau}}{e^{\tau}}
$$

Logo, a função $h(t)$, na equação (19), é nula se, e somente se, $p(\tau)=c$.

Nisso, pode-se afirmar a seguinte relação: se $p\left(r t_{0}\right)=c$, então $t_{0}>A / b$, segue que:

$$
\begin{aligned}
& t_{0}>\frac{A}{b} \Leftrightarrow r t_{0}>r \frac{A}{b} \Leftrightarrow p\left(r t_{0}\right)>p\left(r \frac{A}{b}\right) \Leftrightarrow c>\left(1-e^{r^{2} \frac{A}{b}}+r \frac{A}{b} * e^{r \frac{A}{b}}\right) e^{-r_{\bar{b}}^{A}} \Leftrightarrow c> \\
& \left(e^{-r \frac{A}{b}}-1+r \frac{A}{b}\right) \Leftrightarrow \frac{A r^{2}}{a}>\left(e^{-r^{\frac{A}{b}}}-1+r \frac{A}{b}\right) \Leftrightarrow \frac{A r^{2}}{a}>\left(e^{-r_{\bar{b}} \frac{A}{b}}-1+r \frac{A}{b}\right) \Leftrightarrow
\end{aligned}
$$




$$
\frac{b A r^{2}}{b\left(e^{-r \frac{A}{b}}-1\right)+A r}>a
$$

Dessa forma, concluiu-se que se existe um $t_{0}$, tal que este seja solução da equação (19) e $t_{0}>A / b$, então uma condição para existência deste ponto na equação (19) pode ser expressa pela equação (23).

Por fim, o ponto crítico encontrado - com o software Wolfram Mathematica 8.0, pois se trata de uma função não diferenciável no sentido clássico - é:

$$
t=\frac{T(c)}{r}=\frac{W_{0}\left(-e^{-c-1}\right)+c+1}{r}
$$

Por meio dessa estratégia, é possível determinar o ponto crítico de qualquer ativo físico, uma vez que se possuam os valores de $A, r$ e $a$. Além disso, é importante destacar que na equação (24), $\mathrm{W}_{0}$ representa a parte injetora da função multivalorada W-Lambert (para mais informações sobre esta função ver Corless et al (1996)). Destarte, definiu-se o ponto de mínimo do equipamento no Regime 2.

$\mathrm{Na}$ Figura 4 é possível ver como se comportariam as possíveis curvas da função $A E(t)$.

Figura 4 - Comportamento da função $A E(t)$ no Regime 2

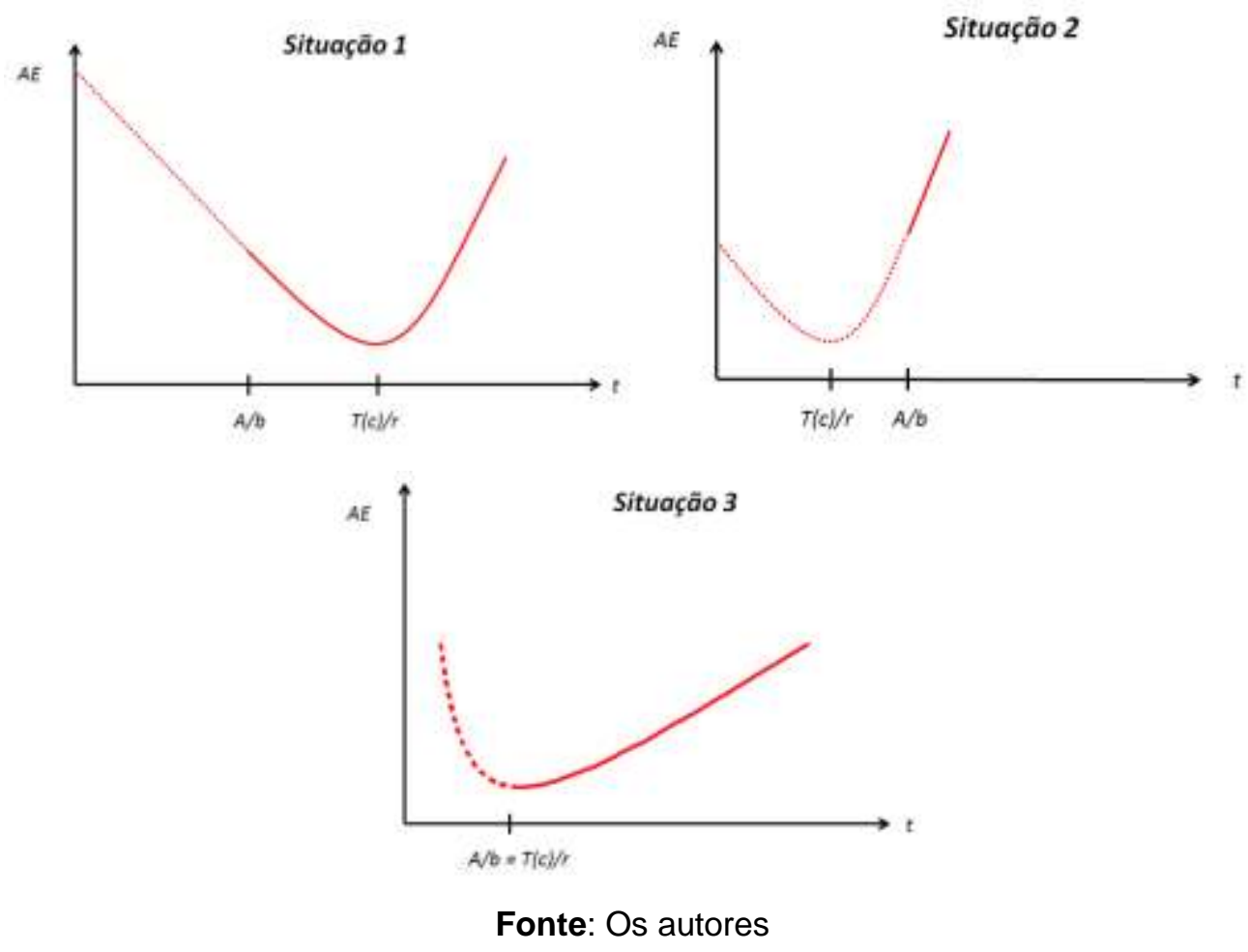

Revista Produção Online. Florianópolis, SC, v. 18, n. 3, p. 850-874, 2018. 
$\mathrm{Na}$ Figura 4, ficam evidenciadas as possíveis curvas da função $A E(t)$ no Regime 2. Na Situação $1, T(c) / r>A / b$. Logo, o ponto de vida econômica está no Regime 2. Por outro lado, na Situação $2, T(c) / r<A / b$. Logo, o mínimo da função não está no Regime 2. Por último, na Situação $3, T(c) / r=A / b$, o ponto crítico do Regime 2 está no ínfimo do intervalo $\left[\frac{A}{b}, \infty[\right.$.

Desse modo, fica definido e demonstrado todo o arcabouço teórico do uso de funções contínuas para modelagem de problemas de substituição de equipamentos. Os autores partiram dos modelos tradicionais discretos utilizados em uma vasta literatura e os transformaram em funções contínuas. Estas funções passaram a ser do tipo não diferenciáveis no sentido clássico. Dessa forma, foi conduzida uma análise não suave das funções formuladas, a fim de obter um método quantitativo com mais rigor dos conceitos de vida econômica e reposição de ativos para a tomada de decisão em problemas de substituição de equipamentos.

\section{ANÁLISE DE RESULTADOS}

Na seção anterior foi feita a modelagem para determinação dos pontos de vida econômica e reposição de ativos para o problema clássico de substituição de equipamentos por meio de funções contínuas e análise não suave. Dessa modelagem é possível determinar dois resultados para os pontos de mínimo no Regime 1 e no Regime 2.

Para o Regime 1, no intervalo [0, $A / b$ [, conclui-se que a função $A E(t)$ possui um comportamento ou crescente ou decrescente. Portanto, o ponto de mínimo da função, pelo Teorema de Weierstrass, encontra-se nos respectivos extremos do intervalo de definição. Pelas equações (25) e (26) é possível calcular os mínimos.

Se a função $A E(t)$ for crescente, então, o seu mínimo é dado pela equação (25) abaixo:

$$
A E(0)=\frac{\left(-1+e^{r}\right)(b+A r)}{r}
$$

Se a função $A E(t)$ for decrescente, então, o seu mínimo é encontrado pela equação (26): 


$$
A E(A / b)=\frac{\left(-1+e^{r}\right)\left(a+\frac{A r(-a+b r)}{b\left(-1+e^{\frac{A r}{b}}\right)}+A r^{2}\right)}{r^{2}}
$$

Já para o Regime 2, na região $\left[\frac{A}{b}, \infty\left[\right.\right.$, se $\frac{b A r^{2}}{b\left(e^{-r \frac{A}{b}-1}\right)+A r}>a$, a equação $A E(t)=$ $\frac{\left(-1+e^{r}\right)}{\left(-1+e^{r t}\right) r^{2}}\left(e^{r t}\left(a+A r^{2}\right)-a(1+r t)\right)$ tem solução para $t$. Uma vez que esta solução depende apenas de $a, r$ e $A$, tal que $c=\frac{A r^{2}}{a}$, por meio da função $T(c)=W_{0}\left(-e^{-c-1}\right)+$ $\mathrm{c}+1$ encontra-se o valor de $t$, tal que $t=\frac{T(c)}{r}$. Além disso, tal solução é única, uma vez que $A E(t)$ é decrescente em $A / b<t<T(c) / r$ e é crescente em $t>T(c) / r$.

Em resumo, até agora se sabe que no Regime 1, o ponto de mínimo se encontra em uma das fronteiras do intervalo $[0, A / b[$, enquanto que no Regime 2 , ou o mínimo se encontra na primeira extremidade, tal que $\frac{T(c)}{r}=A / b$, ou para algum valor $\frac{T(c)}{r}>A / b$. Estes cálculos podem ser facilmente encontrados por meio dos parâmetros iniciais de $a, b, r$ e $A$, os quais representam, respectivamente, a taxa de crescimento do custo de manutenção do equipamento, a taxa de desvalorização do ativo, a taxa mínima de atratividade adotada e o valor de aquisição do equipamento.

\subsection{Análise das lacunas encontradas na revisão de literatura}

Percebe-se que é possível a existência de pontos de mínimo nos dois regimes. Sendo assim, urge que se analise ambos os regimes juntos e se compare os pontos críticos de cada região, a fim de se estabelecer qual o mínimo comum às duas regiões, ou seja, o mínimo global da função. Este ponto será o ponto de vida econômica do ativo. Com essa análise, espera-se responder os questionamentos estabelecidos na Introdução deste trabalho, isto é, (1) se o ponto de vida econômica é único, (2) se é possível existir algum ativo que não possui ponto de vida econômica e, por fim, (3) quais condições podem ser estabelecidas para os parâmetros iniciais do modelo.

Para isso, primeiramente, serão analisados os casos em que a função $A E(t)$ é crescente no Regime 1. Dessa maneira, dado que $A E(0)<A E(T(c) / r)$, então 


$$
\begin{gathered}
\frac{\left(-1+e^{r}\right)(b+A r)}{r}<\frac{\left(e^{r}-1\right)}{r^{2}}\left(a+A r^{2}+a * W_{0}\left(-e^{-\frac{a+A r^{2}}{a}}\right)\right) \Leftrightarrow \\
\frac{r(A r+b)}{a}<1+\frac{A r^{2}}{b}+W_{0}\left(-e^{-1-\frac{A r^{2}}{a}}\right) \Leftrightarrow \\
\frac{A r^{2}}{a}+\frac{b r}{a}<1+\frac{A r^{2}}{a}+W_{0}\left(-e^{-1-\frac{A r^{2}}{a}}\right) \Leftrightarrow \\
\frac{b r}{a}-1<W_{0}\left(-e^{\left.-1-\frac{A r^{2}}{a}\right)} \Leftrightarrow\right. \\
W_{0}^{-1}\left(\frac{b r}{a}-1\right)<W_{0}^{-1}\left(W_{0}\left(-e^{-1-\frac{A r^{2}}{a}}\right)\right) \Leftrightarrow \\
\left(\frac{b r}{a}-1\right) e^{\frac{b r}{a}-1}<-e^{-1-\frac{A r^{2}}{a}} \Leftrightarrow\left(1-\frac{b r}{a}\right) e^{\frac{b r}{a}-1}>e^{-1-\frac{A r^{2}}{a}} .
\end{gathered}
$$

Aplicando ln em ambos os lados da inequação:

$$
\begin{gathered}
\ln \left(\left(1-\frac{b r}{a}\right) e^{\frac{b r}{a}-1}\right)>\ln \left(e^{-1-\frac{A r^{2}}{a}}\right) \Leftrightarrow \\
\ln \left(\left(1-\frac{b r}{a}\right) e^{\frac{b r}{a}-1}\right)>\ln \left(e^{-1-\frac{A r^{2}}{a}}\right) \Leftrightarrow \\
\ln \left(1-\frac{b r}{a}\right)+\frac{b r}{a}>\frac{A r^{2}}{a} .
\end{gathered}
$$

Então, pode-se deixar a inequação em função do parâmetro $A$, multiplicando ambos os lados da inequação por $\frac{a}{r^{2}}$, então:

$$
A>\ln \left(1-\frac{b r}{a}\right)^{-\frac{a}{r^{2}}}-\frac{b}{r}
$$

Logo, a equação (27) é uma condição, em função do parâmetro $A$, para que quando $A E(t)$ for crescente no Regime 1, ou seja, $b r<a$, o mínimo no Regime 1 seja menor que o mínimo no Regime 2. Portanto, é possível listar as seguintes situações de ponto de vida econômica:

- $A<\ln \left(1-\frac{b r}{a}\right)^{-\frac{a}{r^{2}}}-\frac{b}{r}$, se, e somente se, $A E(0)>A E\left(\frac{T(c)}{r}\right)$.

- $A>\ln \left(1-\frac{b r}{a}\right)^{-\frac{a}{r^{2}}}-\frac{b}{r}$, se, e somente se, $A E(0)<A E\left(\frac{T(c)}{r}\right)$;

Revista Produção Online. Florianópolis, SC, v. 18, n. 3, p. 850-874, 2018. 
- $A=\ln \left(1-\frac{b r}{a}\right)^{-\frac{a}{r^{2}}}-\frac{b}{r}$, se, e somente se, $A E(0)=A E\left(\frac{T(c)}{r}\right)$

Para ilustrar essas três situações, foram estabelecidos três possíveis casos de análise de vida econômica.

\subsubsection{Caso 1}

Neste caso, ocorre que $A<\ln \left(1-\frac{b r}{a}\right)^{-\frac{a}{r^{2}}}-\frac{b}{r}$, se, e somente se, $A E(0)>$ $A E\left(\frac{T(c)}{r}\right)$. Logo o mínimo da função $A E(t)$ está em $t=\frac{T(c)}{r}$. Na Figura 5 , há um gráfico indicando o comportamento da função.

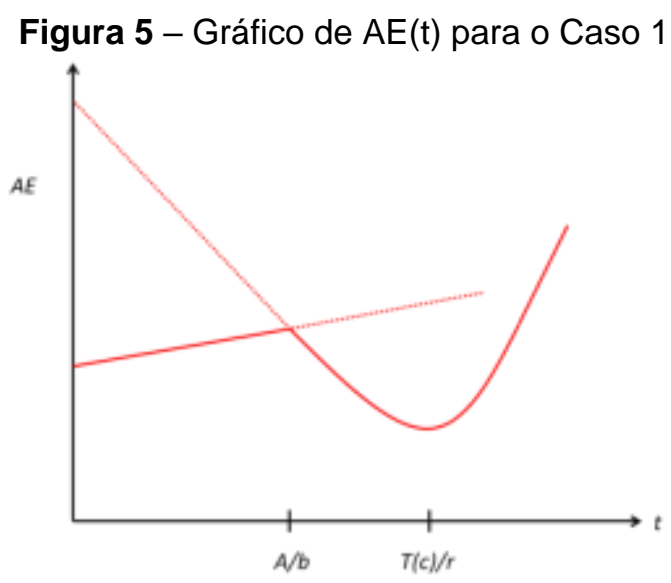

Fonte: Os autores

\subsubsection{Caso 2}

Já para o caso 2, ocorre que $A>\ln \left(1-\frac{b r}{a}\right)^{-\frac{a}{r^{2}}}-\frac{b}{r}$, se, e somente se, $A E(0)<$ $A E\left(\frac{T(c)}{r}\right)$. Logo, o mínimo está em $t=0$, conforme é possível ressaltar na Figura 6 . 


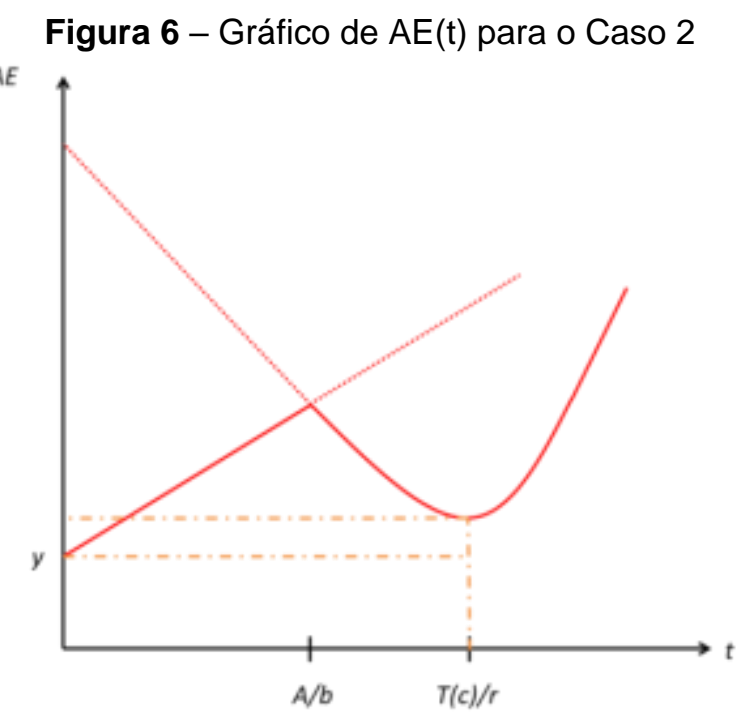

Fonte: Os autores

\subsubsection{Caso 3}

Para o caso 3 , ocorre que $A=\ln \left(1-\frac{b r}{a}\right)^{-\frac{a}{r^{2}}}-\frac{b}{r}$, se, e somente se, $A E(0)=$ $A E\left(\frac{T(c)}{r}\right)$. Logo, o valor mínimo de $A E(t)$ ocorre em ambos os pontos. Na Figura 7 é possível evidenciar como se comporta a função ao longo do tempo.

Figura 7 - Gráfico de $\mathrm{AE}(\mathrm{t})$ para o Caso 3

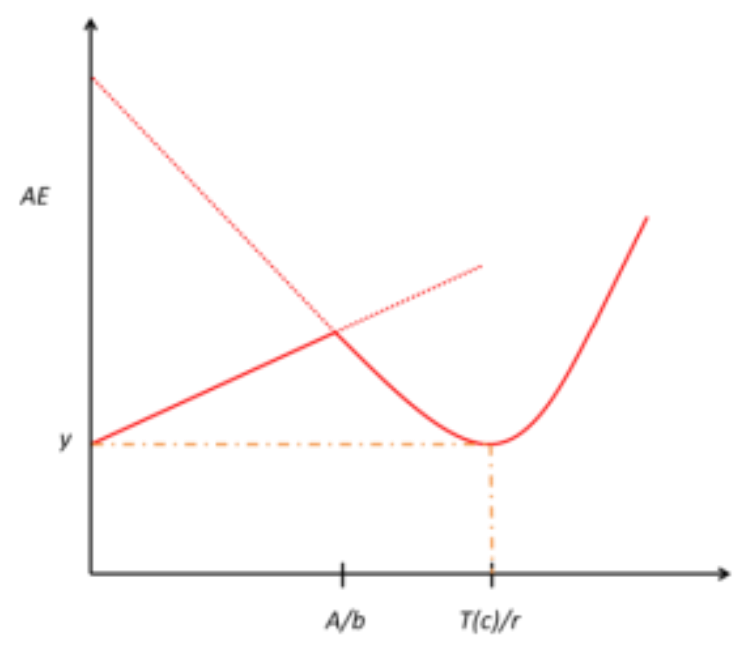

Fonte: Os autores

Para o caso 3, o valor do mínimo é o mesmo nos dois regimes. Contudo, este caso é improvável de ocorrer. Assim como explicado anteriormente na Metodologia, na Figura 3, é possível, de maneira análoga, construir um plano, no qual um eixo é $A$ (lado esquerdo da desigualdade) e o outro eixo é $\ln \left(1-\frac{b r}{a}\right)^{-\frac{a}{r^{2}}}-\frac{b}{r}$ (lado direito da igualdade). Nisso, no plano, há a diagonal como espaço de eventos do Caso 3 , Revista Produção Online. Florianópolis, SC, v. 18, n. 3, p. 850-874, 2018. 
enquanto há um semiplano para o Caso 1 e outro para o Caso 2. Assim, os casos prováveis são apenas o Caso 1 e Caso 2. Portanto, fica demonstrado que o ponto de vida econômica, ou seja, o momento ótimo de substituição de ativos é único. Esta era um dos objetivos deste artigo, conforme estabelecido na Introdução deste trabalho.

Em seguida, buscou-se estabelecer dois casos, nos quais não há solução no Regime 2. Portanto, as soluções estão no Regime 1.

\subsubsection{Caso 4}

No caso 4, conforme explicado na Metodologia, ocorre que $\frac{b A r^{2}}{b\left(e^{\left.-r \frac{A}{b}-1\right)+A r}\right.}<a$, não há uma solução em $t>A / b$. Sendo assim, se $b r<a$, a solução se encontra no Regime 1, a qual é quando $t=0$. Na Figura 8 abaixo, é possível observar como se comporta a função $A E(t)$ neste caso.

Figura 8 - Gráfico de AE(t) para o Caso 4

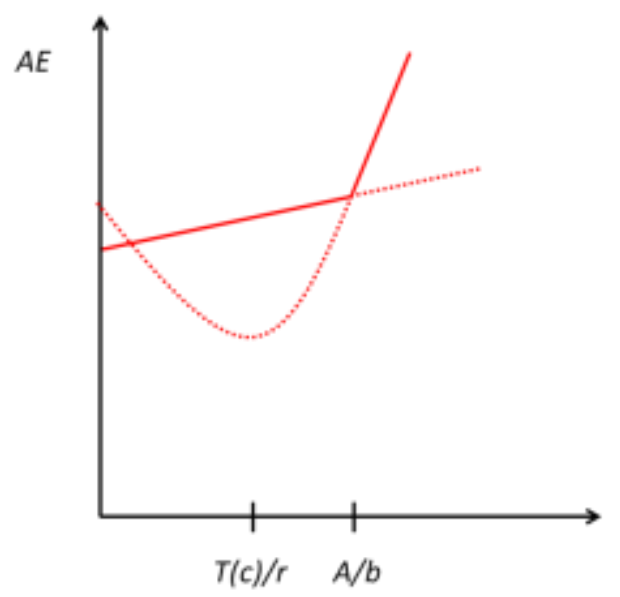

Fonte: Os autores

Percebe-se pela Figura 8 que com um ativo com esta curva de $A E(t)$ o custo equivalente de propriedade só é crescente, de tal forma que não há um momento ótimo para reposição deste ativo. Portanto, tem-se agora a resposta para mais uma das lacunas levantadas na revisão bibliográfica, isto é, se seria possível existir algum equipamento que não seria possível modelar e definir um momento ótimo para sua substituição. 


\subsubsection{Caso 5}

Para o caso 5, de maneira similar ao Caso 4, ocorre que $\frac{b A r^{2}}{b\left(e^{\left.-r^{\frac{A}{b}}-1\right)+A r}\right.}<a$. Contudo, nesse caso a função $A E(t)$ é decrescente, ou seja, $b r>a$. Logo, o mínimo global da função ocorre quando $t=A / b$, conforme é possível visualizar na Figura 9 .

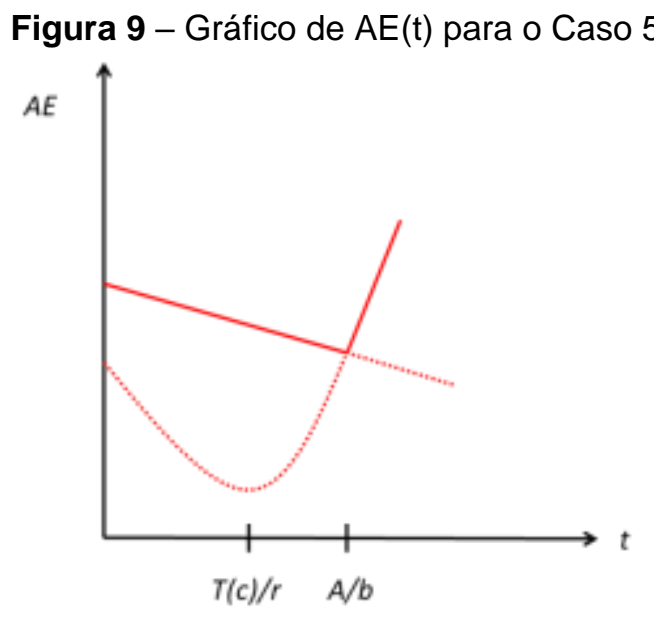

Fonte: Os autores

Além destes casos, é possível estabelecer outro, no qual $A E(t)$ no Regime 1 possui comportamento decrescente e, para o Regime 2, $A E(t)$ admite solução em $t>$ $A / b$.

\subsubsection{Caso 6}

Por último, no caso 6, a função $A E(t)$ é decrescente no Regime 1, ou seja, $b r>$ a. Além disso, para o Regime 2, ocorre que $\frac{b A r^{2}}{b\left(e^{-r \frac{A}{b}}-1\right)+A r}>a$. Logo, o mínimo da função é em $t=\frac{T(c)}{r}$. Na Figura 10, é possível ver o comportamento da função para este caso. 


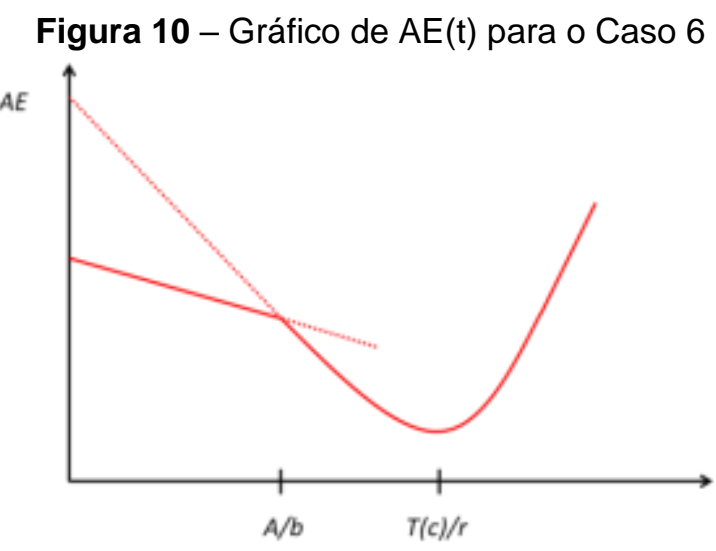

Fonte: Os autores

Em resumo, pela análise de resultados foi possível estabelecer seis diferentes casos para comportamento das curvas de custos de propriedade equivalente por meio da função $A E(t)$ para substituição de ativos. Sendo assim, a metodologia desenvolvida neste artigo conseguiu determinar uma curva de custo equivalente de propriedade para o ativo pela função $A E(t)$, dividida em dois regimes, a qual determina os custos ao longo de cada período do ativo.

Por esses casos, conseguiu-se responder aos questionamentos levantados na Introdução deste trabalho. Pelos casos 1, 2 e 3 foi possível concluir que o ponto de vida econômica é único e pelo caso 4 foi possível concluir que é possível existir um ativo que - dadas as condições iniciais de $a, b, r$ e $A$ - não é possível determinar seu momento ótimo de substituição

Além disso, também foi possível responder à terceira lacuna encontrada na revisão de literatura, isto é, quais as condições podem ser estabelecidas para tomada de decisão de substituição de equipamentos. Assim, estabelece-se que por meio de parâmetros iniciais $a, b, r$ e $A$, os quais representam, respectivamente, a taxa de crescimento do custo de manutenção do equipamento, a taxa de desvalorização do ativo, a taxa mínima de atratividade e valor de aquisição do equipamento, temos as seguintes condições:

(1) com $a>b r$ e $t \geq 0$, se $A>\ln \left(1-\frac{b r}{a}\right)^{-\frac{a}{r^{2}}}-\frac{b}{r}$, o mínimo da função é $A E(0)=$ $\frac{\left(-1+e^{r}\right)(b+A r)}{r}$;

(2) se $A<\ln \left(1-\frac{b r}{a}\right)^{-\frac{a}{r^{2}}}-\frac{b}{r}$, o ponto de mínimo de $A E(t)$ é atingido em $t=\frac{T(c)}{r}$, uma vez que, dada a função $A E(t)$, tal que $c=\frac{A r^{2}}{a}, W_{0}(z)$ é a função $W$ de 
$T(c)=W_{0}\left(-e^{-c-1}\right)+c+1$, têm-se as seguintes possibilidades de solução para $A E(t)$ :

- $a>b r$ e $\frac{b A r^{2}}{b\left(e^{-r_{\bar{b}}^{A}}-1\right)+A r}<a$, se, e somente se, o mínimo de $A E(t)$ é atingido em $t=0$

- $a<b r$ e $\frac{b A r^{2}}{b\left(e^{-r \frac{A}{b}}-1\right)+A r}<a$, se, e somente se, o mínimo de $A E(t)$ é atingido em $t=A / b$;

- $a<b r$ e $\frac{b A r^{2}}{b\left(e^{\left.-r \frac{A}{b}-1\right)+A r}\right.}>a$, se, e somente se, o mínimo de $A E(t)$ é unicamente atingido em $t=T(c) / r$.

\subsection{Aplicação}

Considera-se para aplicação um exemplo de um ativo com os seguintes valores de revenda e manutenção, ambos em função do tempo, conforme a Tabela 1.

Tabela 1 - Valores de manutenção e revenda para um ativo físico

\begin{tabular}{|c|c|c|c|}
\hline Ano & Manutenção & & venda \\
\hline 0 & 0,00 & $\mathrm{R} \$$ & $75.000,00$ \\
\hline 1 & $\mathrm{R} \$ 3.100,00$ & $\mathrm{R} \$$ & $52.000,00$ \\
\hline 2 & $R \$ \quad 6.200,00$ & $\mathrm{R} \$$ & $29.000,00$ \\
\hline 3 & $\mathrm{R} \$ 9.300,00$ & $\mathrm{R} \$$ & $6.000,00$ \\
\hline 4 & $\mathrm{R} \$ 12.400,00$ & $\mathrm{R} \$$ & . \\
\hline 5 & $R \$ 15.500,00$ & $\mathrm{R} \$$ & - \\
\hline 6 & $\mathrm{R} \$ 18.600,00$ & $\mathrm{R} \$$ & . \\
\hline 7 & $\mathrm{R} \$ 21.700,00$ & $\mathrm{R} \$$ & . \\
\hline 8 & $\mathrm{R} \$ 24.800,00$ & $\mathrm{R} \$$ & - \\
\hline 9 & $\mathrm{R} \$ 27.900,00$ & $\mathrm{R} \$$ & 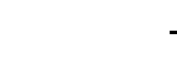 \\
\hline 10 & $\mathrm{R} \$ 31.000,00$ & $\mathrm{R} \$$ & - \\
\hline
\end{tabular}

Pela Tabela 1, tem-se o custo de aquisição $(A)$ igual à $\mathrm{R} \$ 75.000,00$, a taxa de desvalorização $(b)$ à $R \$ 23.000,00$, o coeficiente da função de custo de manutenção (a) (por regressão linear simples) à $\mathrm{R} \$ 3.100,00$. Além disso, considera-se como dado pelo problema a taxa de desconto $(r)$ de $10 \%$. 
Dessa maneira, como $a>b r$, então $A E(t)$ é crescente no Regime 1. Além disso, como $\frac{b A r^{2}}{b\left(e^{-r \frac{A}{b}}-1\right)+A r}>a$, há ponto crítico no Regime 2. Mais ainda, dado que $A<$ $\ln \left(1-\frac{b r}{a}\right)^{-\frac{a}{r^{2}}}-\frac{b}{r}$, o mínimo global da função está no Regime 2. Na sequência, aplicando os valores encontrados de $a, b, r$ e $A$, é possível determinar que, por meio da equação (24) $t=\frac{T(c)}{r}=\frac{W_{0}\left(-e^{-c-1}\right)+c+1}{r}$, a vida econômica ocorre no ano oito com valor de $\mathrm{R} \$ 25.644,65$. Na Figura 11 é possível visualizar o comportamento da função $A E(t)$ para este ativo.

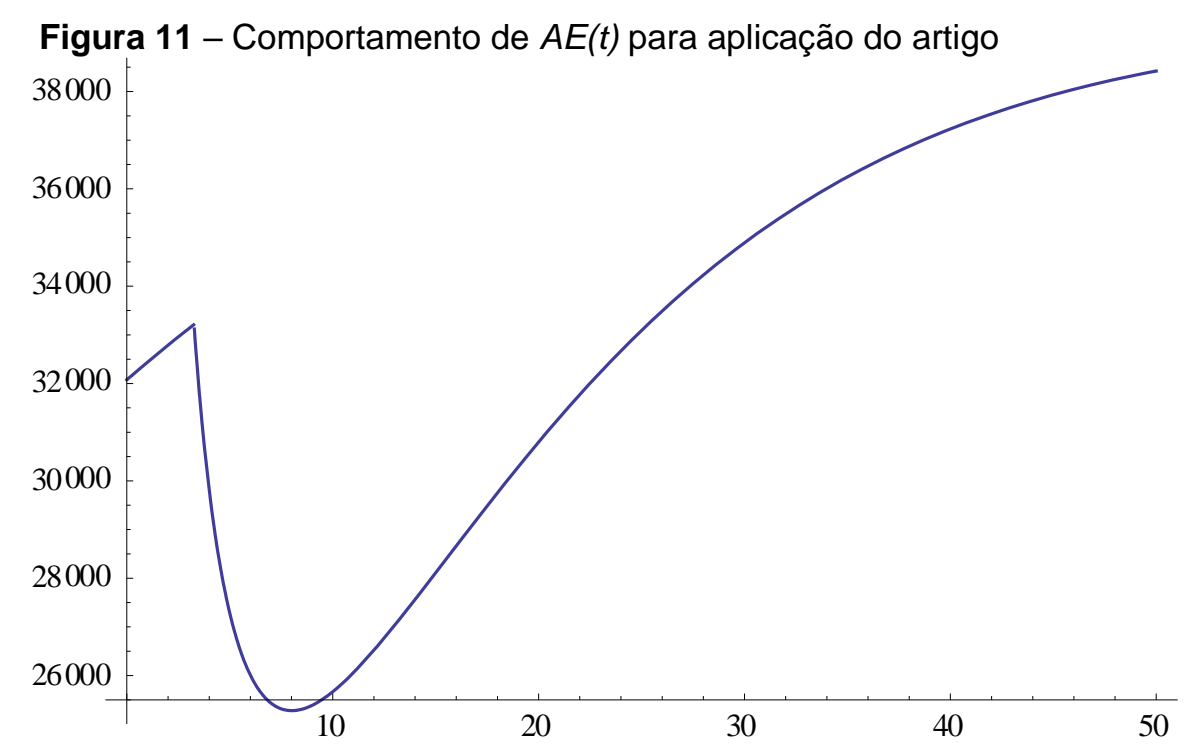

Fonte: Os autores

Na Figura 11 é possível ver que nos primeiros anos a curva de custo de propriedade do ativo é crescente. Depois, a partir do ano quatro, a curva se torna decrescente, atingindo um mínimo; isto é, a vida econômica, no ano oito, cujo valor é de $\mathrm{R} \$ 25.644,65$. Após este ano, o custo de propriedade volta a crescer.

\section{CONSIDERAÇÕES FINAIS}

As metodologias tradicionais aplicadas nos problemas substituição de equipamentos são modelagem por programação dinâmica e análise de fluxo de caixa, tal que ambas as abordagens utilizam modelos discretos. Nesse sentido, verificou-se que há lacunas sobre as condições de existências e unicidades dos pontos de vida econômica e momentos ótimos de reposição de ativos. Mais especificamente, (1) não 
foram encontrados na literatura trabalhos sobre a possibilidade de existir mais de um ponto de vida econômica, isto é, não foi demonstrado se é possível existir mais de um momento ótimo de substituição. Além disso, (2) também não foi encontrado nenhum trabalho sobre a possibilidade de existir algum ativo físico que nunca seria substituído, isto é, sua curva de custo equivalente de propriedade não atinge um ponto mínimo.

Consequentemente, os autores deste trabalho tiveram como objetivo preencher tais lacunas por meio de um novo modelo para tomada de decisão em substituição de equipamentos. Assim, iniciou-se a partir dos modelos tradicionais discretos de fluxo de caixa, os quais foram transformados em funções contínuas. Estas funções passaram a ser do tipo não diferenciáveis no sentido clássico. Dessa forma, foi conduzida uma análise não suave das funções formuladas, a fim de obter um método quantitativo com mais rigor dos conceitos de vida econômica e reposição de ativos para a tomada de decisão em problemas de substituição.

Pelos resultados obtidos, foi demonstrado que, para o problema de substituição de equipamentos, há um único ponto de vida econômica, isto é, um único momento ótimo para substituição de equipamentos. Mais ainda, foi também demonstrado que é possível existir um modelo para um ativo que não atingirá um momento ótimo para reposição. Este será o caso de um ativo com curva de custo equivalente de propriedade crescente.

Por fim, foi possível formular um modelo para calcular o ponto de mínimo na curva de custo equivalente de propriedade, ou seja, o momento ótimo de substituição de equipamentos. Isso ocorreu por meio dos parâmetros iniciais do modelo: (1) taxa de crescimento do custo de manutenção do equipamento, (2) valor de aquisição, (3) taxa de desvalorização e (4) taxa mínima de atratividade.

\section{REFERÊNCIAS}

ABENSUR, Eder Oliveira. Replacement of capital goods: an optimization model from the perspective of Production Engineering. Gestão \& Produção, 2015.

http://dx.doi.org/10.1590/0104-530X1690-14

ADKINS, Roger; PAXSON, Dean. Deterministic models for premature and postponed replacement. Omega, v. 41, n. 6, p. 1008-1019, 2013.

https://doi.org/10.1016/j.omega.2013.01.002

ALCHIAN, Armen. Economic replacement policy. An abbreviated version of $r-224$. Rand Corp Santa Monica Calif, 1958. 
BELLMAN, Richard. Equipment replacement policy. Journal of the society for industrial and applied Mathematics, v. 3, n. 3, p. 133-136, 1955. https://doi.org/10.1137/0103011

BROWN, Robert J.; YANUCK, Rudolph R.: Introduction to Life Cycle Costing. The Fairmont Press, Inc. e Prentice-Hall, Inc. EUA, 1985.

BUSER, Stephen A.: Laplace Transforms as Present Value Rules: A Note. The Journal of Finance, Vol. 41, No. 1 (Mar., 1986), pp. 243-247. http://10.1111/j.15406261.1986.tb04502.x

CESCA, Igor G.: Custo de ciclo de vida de equipamentos industriais com programação dinâmica. Sinergia (IFSP. Online), v. 18, p. 30-34, 2017.

CORLESS, R. M.; GONNET G. H.; HARE D. E. G.; JEFFREY, D. J.; KNUTH, D. E.: On the Lambert W Function, Adv. Comput. Math., no. 4, 329-359, 1996.

DHILLON, B. S.: Life Cycle Costing for Engineers. CRC Press, 2010.

FERNANDEZ, Viviana P. Observable and unobservable determinants of replacement of home appliances. Energy Economics, v. 23, n. 3, p. 305-323, 2001.

https://doi.org/10.1016/S0140-9883(00)00066-9

HARTMAN, Joseph C. Multiple asset replacement analysis under variable utilization and stochastic demand. European Journal of Operational Research, v. 159, n. 1, p. 145-165, 2004. https://doi.org/10.1016/S0377-2217(03)00397-7

LONGO, M.; ZANOBETTI, D. Criteria for asset replacement studies. Engineering Costs and Production Economics, v. 17, n. 1, p. 271-275, 1989. https://doi.org/10.1016/0167188X(89)90076-1

MAUER, David C.; OTT, Steven H. Investment under uncertainty: The case of replacement investment decisions. Journal of Financial and Quantitative Analysis, v. 30, n. 4, p. 581605, 1995. https://doi.org/10.2307/2331278

MARQUES, Gláucio M.: Transformação e Substituição de Equipamentos Utilizando Equações Diferenciais e Programação Dinâmica. Dissertação de mestrado, Universidade Federal de Viçosa, MG, 2003.

NAKAGAWA, Toshio. A summary of discrete replacement policies. European Journal of Operational Research, v. 17, n. 3, p. 382-392, 1984. https://doi.org/10.1016/03772217(84)90134-6

PARK, Chan S. Contemporary engineering economics. Upper Saddle River, NJ: Prentice Hall, 2002.

RIECHI, Jorge Luiz; TORMOS, Bernardo; HILLEBRAND, Marcos Vinicius Jacometo. Otimização dos custos de frota urbana com uso de modelo combinado de life cycle cost $\mathrm{e}$ simulação de Monte Carlo. Revista Produção Online, v. 17, n. 2, p. 667-691, 2017. http://dx.doi.org/10.14488/1676-1901.v17i2.2627

ROSS, Stephen; WESTERFIELD, Randolph W.; JAFFE, Jeffrey F.: Administração Financeira. Traduzido de Corporate Finance, 2nd Ed. Editora Atlas, 2002.

SULLIVAN, William G.; MCDONALD, Thomas N.; VAN AKEN, Eileen M. Equipment replacement decisions and lean manufacturing. Robotics and Computer-Integrated

Revista Produção Online. Florianópolis, SC, v. 18, n. 3, p. 850-874, 2018. 
Manufacturing, v. 18, n. 3, p. 255-265, 2002. https://doi.org/10.1016/S0736-5845(02)00016$\underline{9}$

WADDELL, R.: A Model for Equipment Replacement Decisions and Policies. Interfaces, Vol. 13, No. 4 (Aug., 1983), pp. 1 - 7. https://doi.org/10.1287/inte.13.4.1

YATSENKO, Yuri; HRITONENKO, Natali. Machine replacement under evolving deterministic and stochastic costs. International Journal of Production Economics, v. 193, p. 491-501, 2017. https://doi.org/10.1016/j.ijpe.2017.08.013

YE, Meng-Hua. Optimal replacement policy with stochastic maintenance and operation costs. European Journal of Operational Research, v. 44, n. 1, p. 84-94, 1990.

https://doi.org/10.1016/0377-2217(90)90317-5

ZAMBUJAL-OLIVEIRA, João; DUQUE, João. Operational asset replacement strategy: A real options approach. European Journal of Operational Research, v. 210, n. 2, p. 318-325, 2011. https://doi.org/10.1016/j.ejor.2010.09.011

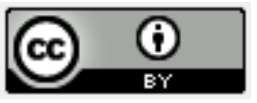

Artigo recebido em 14/08/2017 e aceito para publicação em 07/12/2017 DOI: http://dx.doi.org/10.14488/1676-1901.v18i3.2977 